This is an Open Access article, distributed under the terms of the Creative Commons Attribution licence (http://creativecommons.org/licenses/by/4.0/), which permits unrestricted re-use, distribution, and reproduction in any medium, provided the original work is properly cited.

doi:10.1017/jfm.2019.806

\title{
Diffusive growth of successive bubbles in confinement
}

\author{
Álvaro Moreno Soto ${ }^{1,2, \dagger}$, Detlef Lohse ${ }^{1}$ and Devaraj van der Meer ${ }^{1, \dagger}$ \\ ${ }^{1}$ Physics of Fluids Group and Max Planck Center Twente, MESA+ Institute and J. M. Burgers Centre \\ for Fluid Dynamics, Faculty of Science and Technology, University of Twente, P.O. Box 217, \\ 7500 AE Enschede, The Netherlands \\ ${ }^{2}$ Department of Mechanical Engineering, Massachusetts Institute of Technology, 77 Massachusetts \\ Avenue, Cambridge, Massachusetts 02139, USA
}

(Received 25 January 2019; revised 30 September 2019; accepted 2 October 2019)

We analyse how a succession of single bubbles extracts dissolved gas from a liquid solution while they grow and detach in a confinement induced by the presence of lateral walls. Like bubbles growing on a liquid-immersed unconfined surface, these bubbles absorb the dissolved gas in the liquid around them and hence deplete their surroundings. The supersaturation level, $\zeta$, stands out as the main parameter which determines the diffusive bubble dynamics, both in the confined and unconfined scenarios. For slightly supersaturated solutions, the bubble evolution is rather similar for the two cases. We observe nonetheless mildly higher concentration gradients within confinement due to the lack of gas renewal. This causes a slightly enhancement of density-driven convection as compared to the unconfined case, which results in a higher mass transfer rate towards the bubble and a somewhat faster long-term gas depletion. For larger supersaturations, the onset of natural convection is inhibited by the presence of the confinement. Confinement promotes the gas mixing within the cavity as well. These two effects combined result in a slower depletion in the confined case as compared to the unconfined one. The two opposite behaviours for small and large supersaturation suggest that there must be a transition in between the two scenarios. The cross-over has been estimated to occur at $\zeta \approx 0.17$. We propose a modified depletion model which accounts for the confined configuration and its effect on the effective area through which gas diffuses into the bubble. The model can accurately describe the experimental results and sheds more light on the origin of the depletion effect due to the successive bubble growth.

Key words: bubble dynamics, buoyant boundary layers, convection in cavities

\section{Introduction}

Bubbles have been studied from many different points of view since their behaviour is crucial to a wide variety of applications. They play a critical role in chemical

$\dagger$ Email addresses for correspondence: a.morenosoto@utwente.nl, d.vandermeer@utwente.nl 
reacting processes, such as electrolysis (Verhaart, de Jonge \& van Stralen 1980; Sillen et al. 1982) and catalysis (Oehmichen, Datsevich \& Jess 2010; Somorjai \& $\mathrm{Li}$ 2010). Their generation is extremely important in the confinement of nano- and micro-channels (Barber et al. 2010; Mukherjee, Kandlikar \& Edel 2011; Bao et al. 2017) since bubbles are known to (partially) block them and impede their proper use. Bubbles in a flow travelling through a conduit are formed either of dissolved gas, which accumulates in cracks along the walls, or liquid vapour originating from boiling or chemical processes occurring along the channel (Ajaev \& Homsy 2006; Gedupudi et al. 2011; Zu et al. 2011). When a bubble partially blocks a conduit, the flow needs to circumvent the bubble surface and consequently the flow rate decreases since the cross-section is reduced. Hence, the mass transfer towards the bubble is altered and the bubble shape is modified ( $\mathrm{Zu}$ et al. 2011; Bao et al. 2017). Ultimately, bubbles can grow up to a point that they fully block the channel (Hong et al. 2016), impeding mass transport and causing clogging. In these cases, heat (Barber et al. 2010; Yin \& Jia 2016) and mass transfer considerations are essential to understand bubble growth (Evans \& Machniewski 1999). When the growth of the bubble is governed by diffusion, in principle the Epstein \& Plesset (1950) theory still applies; nonetheless, several modifications need to be adopted to account for the inertial terms corresponding to the flow running through the channels (Leighton 2011).

In this article, we deal with a geometrical configuration similar to the confinement induced in the previously mentioned nano-/micro-channels: a series of single bubbles grow in succession in a domain constrained by lateral walls, an intermediate step between bubbles growing on a liquid-immersed unconfined surface and full confinement. In the latter case, bubbles grow in a volume which cannot be deformed and therefore their growth implies the compression of the liquid surrounding it (Vincent \& Marmottant 2017). In this scenario, the system can be approximated as a harmonic oscillator and the original theory of Epstein \& Plesset (1950) needs to be adapted accordingly. As for bubbles growing on an unconfined surface, the recent work of Moreno Soto et al. (2017) showed that such bubbles deplete their surroundings as they diffusively grow and absorb the dissolved gas in the liquid solution. The local decrease of gas concentration results in a decrease in the solution density, which promotes the onset of (natural) density-driven convection from the middle stages of the bubble growth, therefore enhancing the mass transfer (Moreno Soto et al. 2019). As a consequence, bubbles absorb more gas than diffusion through the bulk alone can supply. The subsequent bubbles grow in a region which has less available gas in dissolution, hence their growth rate is significantly slowed down.

A deeper understanding of how bubbles behave in confined domains with no external forced flows involved is necessary to understand the fundamentals of bubble growth in nano- and micro-channels. With the presence of confinement around a succession of bubbles growing on a surface we intend to shed more light on the origin of depletion and gain a better understanding of the way in which bubbles absorb the gas from their surroundings. Our system remains in a quasi-static configuration and, thus, the bubbles grow mainly by diffusion (Epstein \& Plesset 1950).

Along this article, we first introduce the experimental set-up (\$2) and summarise earlier results regarding the analysis of the bubble growth dynamics ( $\$ 3)$. Afterwards, we focus particularly on two independent scenarios: in the first, we study the different bubble growth evolution in confinement for different supersaturation levels $\zeta(\S 4)$; in the second, we compare the successive growth of bubbles in confinement with that of bubbles on an unconfined surface $(\S 5)$. Finally, $\S 6$ contains the concluding remarks. 
(a)

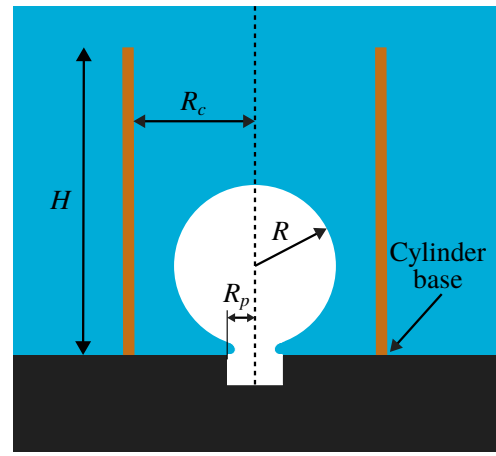

(b)

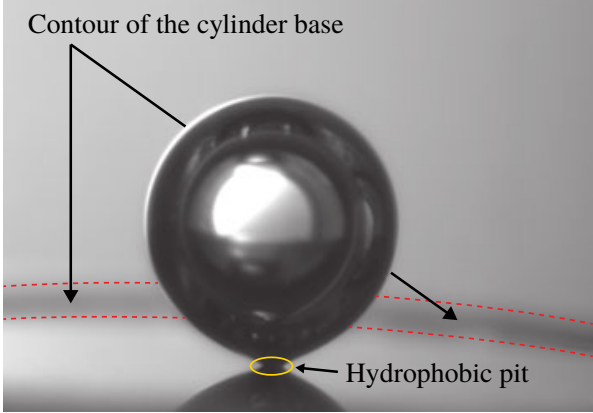

FIGURE 1. (Colour online) (a) Schematic (not to scale) of the experimental geometry with the substrate holding the cylindrical lateral confinement of radius $R_{c}=2.5 \mathrm{~mm}$ and height $H=10 \mathrm{~mm}$ around the growing bubble. The single bubble grows from the superhydrophobic pit of radius $R_{p}=50 \mu \mathrm{m}$ according to the imposed supersaturation level $\zeta$. Once the bubble detaches, a subsequent one nucleates and the succession continues. (b) Snapshot of one of the experiments. The contour of the cylinder base surrounding the bubble can be appreciated. The position of the superhydrophobic pit from which the bubble nucleates and grows is also indicated. One clearly detects the neck joining the entrapped gas in the pit and the bubble growing outside (Moreno Soto et al. 2017).

\section{Experimental set-up and procedure}

The experiments were performed in a pressure-controlled set-up. For details concerning the fabrication and use of the equipment, the reader is referred to Enríquez et al. (2013). To summarise, a mixing tank contained a mixture of liquid (ultra-pure water) and gas $\left(\mathrm{CO}_{2}\right)$ saturated at a certain pressure $P_{0} \approx 9$ bars and temperature $T \approx 20^{\circ} \mathrm{C}$. The water was degassed by means of a vacuum pump prior to experiments to ensure that there were no other spurious gases in solution. The other (measurement) tank contained a silicon substrate which was held by a lateral clip. A preferential spot for bubbles to nucleate and grow was etched on its surface and was composed of black silicon (Stubenrauch et al. 2006; Borkent et al. 2009; Liu et al. 2014), a superhydrophobic material. Around this site (a pit of radius $R_{p}=50 \mu \mathrm{m}$ ), a glass cylinder of radius $R_{c}=2.5 \mathrm{~mm}$ and height $H=10 \mathrm{~mm}$ was glued to the substrate by means of curing a hydrophilic resin with an ultraviolet light. A schematic of the set-up can be seen in figure 1, together with an experimental snapshot.

At the beginning of the experiment, the measurement tank was pressurised with $\mathrm{CO}_{2}$ gas at the same saturation pressure $P_{0}$ as the mixing tank. Then, by means of different valves, the solution was brought from the main tank to partially fill the experimental one. To generate bubbles, we decreased the pressure inside the latter to a value $P_{s}<P_{0}$, and therefore, the solution became supersaturated and bubbles began to grow from the hydrophobic pit. Once a bubble had detached, a new subsequent bubble formed and the succession continued. The time between detachment and nucleation of the subsequent bubble was larger than $10 \mathrm{~s}$, such that no advective flow related to the detachment and rise of the previous bubble (which is typically dissipated within $1 \mathrm{~s}$ ) were expected to affect the growth of the subsequent one (Moreno Soto et al. 2017).

We performed different sets of experiments starting from the same saturation pressure $P_{0} \approx 9$ bars. The supersaturation level $\zeta=\left(c_{0}-c_{s}\right) / c_{s}$ (where $c_{0}$ and $c_{s}$ are the bulk concentration and the saturation concentration at $P_{s}$, respectively) can 
be written in terms of pressure by using Henry's law, $c=k_{H}(T) P$, where in general $k_{H}(T)$ is a decreasing function of temperature dependent on the gas/liquid couple. By decreasing the pressure to different values of $P_{s}$ and keeping the temperature constant at all times, we achieved different values of $\zeta=\left(P_{0}-P_{s}\right) / P_{s} \approx 0.15,0.25$ and 0.47 .

The cylinder curvature may affect the acquisition of images since the light illuminating the experiments is refracted while crossing the cylinder surface. We have made use of a ray tracer (Huisman, van Gils \& Sun 2012) to confirm that the refraction resulting from the light crossing the cylinder walls was inconsequential, and hence, no correction to the images needed to be applied.

As bubbles approached their detachment radius, due to the effect of buoyancy their shape started to become that of a prolate spheroid. In order to calculate a precise bubble radius, we have compared three different techniques: in the first, we fit a circle to the bubble contour; in the second, the radius was obtained by equating the measured projected area of the full bubble to that of a perfect circle; in the third, we computed the volume of the revolution body generated by rotating the bubble profile around its vertical symmetry axis. We found that, within measurement error, the three methods produced identical outcomes except for the very last moments close to bubble detachment. The variability was, however, less than $1 \%$, thus we could safely assume that the bubbles were perfectly spherical at all times.

\section{Analysis of the growth dynamics: theoretical framework}

Bubble growth in mildly supersaturated solutions is standardly associated with diffusion-driven mass transfer phenomena. In this section, we summarise results from Enríquez et al. (2014) and Moreno Soto et al. (2017, 2019), where the behaviour of single bubbles growing on a substrate immersed in a mildly supersaturated solution is described in depth. To study the behaviour of bubbles in such a configuration, two dimensionless quantities are defined:

$$
\epsilon=\frac{R}{R_{0}}, \quad x=\sqrt{\left(\frac{2 D \zeta c_{s}}{\rho_{g} R_{0}^{2}}\right) t},
$$

where $R$ is the bubble radius, $R_{0}$ is a reference radius which is taken to be the radius of the pit $R_{p}, D \approx 1.77 \times 10^{-9} \mathrm{~m}^{2} \mathrm{~s}^{-1}$ is the diffusion coefficient of $\mathrm{CO}_{2}$ in water and $\rho_{g}$ is the gas density. Here, $\epsilon$ represents a dimensionless bubble radius and $x$ a dimensionless square root of time. The diffusive bubble growth is then characterised by the Epstein \& Plesset (1950) equation slightly modified to account for the presence of the substrate below the bubble. The asymptotic solution then becomes

$$
\epsilon \approx\left(\sqrt{\frac{\zeta c_{s}}{2 \pi \rho_{g}}}+\sqrt{\frac{1}{2}+\frac{\zeta c_{s}}{2 \pi \rho_{g}}}\right) x \equiv S^{*} x .
$$

As one can deduce from this equation, the bubble growth is strongly dependent on the supersaturation level $\zeta$, which will be our control parameter in the experiments provided below.

From the middle stages of the bubble growth, an onset to density-driven convection takes place and enhances the mass transfer towards the bubble. Two characteristic dimensionless numbers, namely the Sherwood number $S h$ and the Rayleigh number $R a$, are then defined. The former represents the dimensionless total mass transfer 
towards the bubble, whereas the latter relates buoyancy to viscous and diffusive effects. These two numbers can be written as (see e.g. Clift, Grace \& Weber 1978; Bejan 1993)

$$
S h=\frac{2 \rho_{g} R \dot{R}}{D\left(c_{0}-c_{s}\right)}, \quad R a=\frac{g \lambda_{c}\left(c_{0}-c_{s}\right)(2 R)^{3}}{\nu D},
$$

where $\lambda_{c}$ is the concentration expansion coefficient of the $\mathrm{CO}_{2}$-water solution (with a typical value of $\approx 6.6 \times 10^{-4} \mathrm{~m}^{3} \mathrm{~kg}^{-1}$ ) and $v$ the kinematic viscosity. The onset of convection originates from the density decrease in the solution around the bubble as it absorbs the dissolved gas and depletes the liquid. Therefore, the parameter $\lambda_{c}$ becomes very relevant since it relates the way in which a solution changes in density to the concentration of dissolved gas. In the case of $\mathrm{CO}_{2}$, a growing bubble may then cause a relevant decrease in the density of the surrounding liquid, which causes the formation of a buoyant depleted concentration boundary layer around the bubble and a significant enhancement of mass transfer, i.e. it promotes the transition to densitydriven convection during the diffusive bubble growth. For the case of a sphere with fixed radius in steady-state (natural) convection in an infinite medium, $S h$ and $R a$ can be related as (see e.g. Bejan 1993)

$$
S h=2+K R a^{1 / 4},
$$

where 2 is the value of $S h$ in the pure diffusive case and $K \approx 0.569$ is a fitting constant (Bergman et al. 2011; Rahman 2013).

For the study of the long-term depletion as the bubble succession continues within the cylinder, the depletion number is defined as

$$
\Upsilon_{n}=\frac{\tilde{c}_{n}-c_{s}}{c_{0}-c_{s}}
$$

where $n$ is the index indicating the bubble number within the succession and $\tilde{c}_{n}$ is the local apparent infinite concentration that the $n$th bubble perceives due to the decreased gas concentration resulting from the previous bubble departure. It gives an estimation of how much gas is transported away by a bubble upon detachment due to the enhanced mass transfer rate towards it, which cannot be compensated by the gas diffusion through the bulk. Consequently, the local apparent concentration that subsequent bubbles experience becomes smaller and smaller. By means of this description, we redefine (3.3) to consider the effects of depletion as

$$
\widetilde{S h}=\frac{2 \rho_{g} R \dot{R}}{D\left(\tilde{c}_{n}-c_{s}\right)}=\frac{S h_{n}}{\Upsilon_{n}}, \quad \widetilde{R a}=\frac{g \lambda_{c}\left(\tilde{c}_{n}-c_{s}\right)(2 R)^{3}}{\nu D}=R a_{n} \Upsilon_{n} .
$$

The curves $S h_{n}$ versus $R a_{n}$ can then be collapsed onto one universal curve $\widetilde{S h}=f(\widetilde{R a})$ from which $\Upsilon_{n}$ is obtained. Here, $\Upsilon_{n}$ can be analytically estimated by adapting the solution of a pure diffusive problem to account for the effect of convection caused by the density difference and of the refreshing flows originating at the detachment and subsequent rise of the bubble (which has been determined to be small in comparison). We note again that the knowledge presented until now has been previously discussed in detail in previous works. The main new analysis that we present in this article is the effect of lateral confinement on a succession of bubbles. 
As a bubble grows and absorbs gas from its surroundings, a concentration boundary layer develops following $\sim \sqrt{D t}$. The typical time needed for this diffusive boundary layer to occupy the full width of the cylinder can be estimated as $R_{c}^{2} / D \approx 60$ min, whereas it takes $H^{2} / D \approx 940$ min to cover its full length. Bearing in mind that this is a very conservative estimation and that our experiments run for $\approx 240 \mathrm{~min}$, we can safely presume that, in the long term, the diffusive boundary layer occupies the full width of the cylinder and can only evolve in the vertical direction towards the aperture of the confinement (which does not affect the physics studied here). It is known that this layer reaches a much longer length than the bubble radius as the succession continues (Moreno Soto et al. 2017). In that scenario, the bubble can be approximated as a plane sink which extracts gas from a certain bulk. In view of the above discussion, it is reasonable to assume that the problem becomes one-dimensional, a hypothesis which has been very recently corroborated by Rivero-Rodríguez \& Scheid (2019). In their work, they prove that, under regimes characterised by a very small Péclet number $P e$, i.e. dominated by diffusion, the concentration field far away from the bubble only evolves in the axial direction of the confinement. The discrete depletion number reads thus (for a complete derivation, refer to appendix A):

$$
\begin{aligned}
\Upsilon_{n}= & \Upsilon_{n-1}-\frac{4 \rho_{g} R_{d e t}^{3}}{3 R_{c}^{2} t_{d e t} D\left(c_{0}-c_{s}\right)}\left[2 \sqrt{\frac{D t_{d e t}}{\pi}} \exp \left(-\frac{\left(R_{d e t}+\alpha \sqrt{3.3 D t_{d e t}}\right)^{2}}{4 D t_{d e t}}\right)\right. \\
& \left.-\left(R_{d e t}+\alpha \sqrt{3.3 D t_{d e t}}\right) \operatorname{erfc}\left(\frac{R_{d e t}+\alpha \sqrt{3.3 D t_{d e t}}}{2 \sqrt{D t_{d e t}}}\right)\right],
\end{aligned}
$$

where $R_{\text {det }} \approx 715 \mu \mathrm{m}$ and $t_{\text {det }}$ are the corresponding radius (Fritz 1935; Og̃uz \& Prosperetti 1993; Moreno Soto et al. 2017) and experimentally measured time at detachment, and $\alpha \in[0,1]$ is a fitting constant which relates to the distance within the concentration boundary layer (where 0 corresponds to the bubble interface and 1 constitutes the limit of the boundary layer) that defines the apparent bulk concentration for the subsequent bubble in the succession and, consequently, is a decreasing function of $\zeta$, i.e. bubbles deplete their surroundings faster with increasing $\zeta$ (Moreno Soto et al. 2019). Whereas $R_{\text {det }}$ is a parameter which is determined by a force balance and remains almost constant throughout our experiments, the measurement of the detachment time $t_{d e t}$ and $\alpha$ incorporates convective effects as the bubble grows. Indeed, $t_{d e t}$ increases for subsequent bubbles as the effect of depletion becomes more significant. Note that (3.7) differs slightly from the expression provided in Moreno Soto et al. (2017), where a spherically symmetric three-dimensional situation was modelled, and that the concentration gradient referred to in the following corresponds to the one measured in the longitudinal axis of the cylinder. Towards the lowest limit in which $R_{c}=R_{d e t}$, the model in (3.7) is expected to become increasingly precise, since the assumption of a one-dimensional scenario becomes valid at progressively earlier times as $R_{c}$ approaches $R_{\text {det }}$. This could unfortunately not be tested experimentally due to the limitations of our set-up. In addition, the confinement is not expected to play a significant role when $R_{c} \gg R_{d e t}$ and the problem can be solved as in Moreno Soto et al. (2017).

\section{Effect of the supersaturation level $\zeta$ in confined bubble growth}

In $\S 3$, we discussed that the supersaturation level $\zeta$ determines the speed at which bubbles grow, as one can see from the definitions of $x$ and $S^{*}$ in (3.2). The higher its 

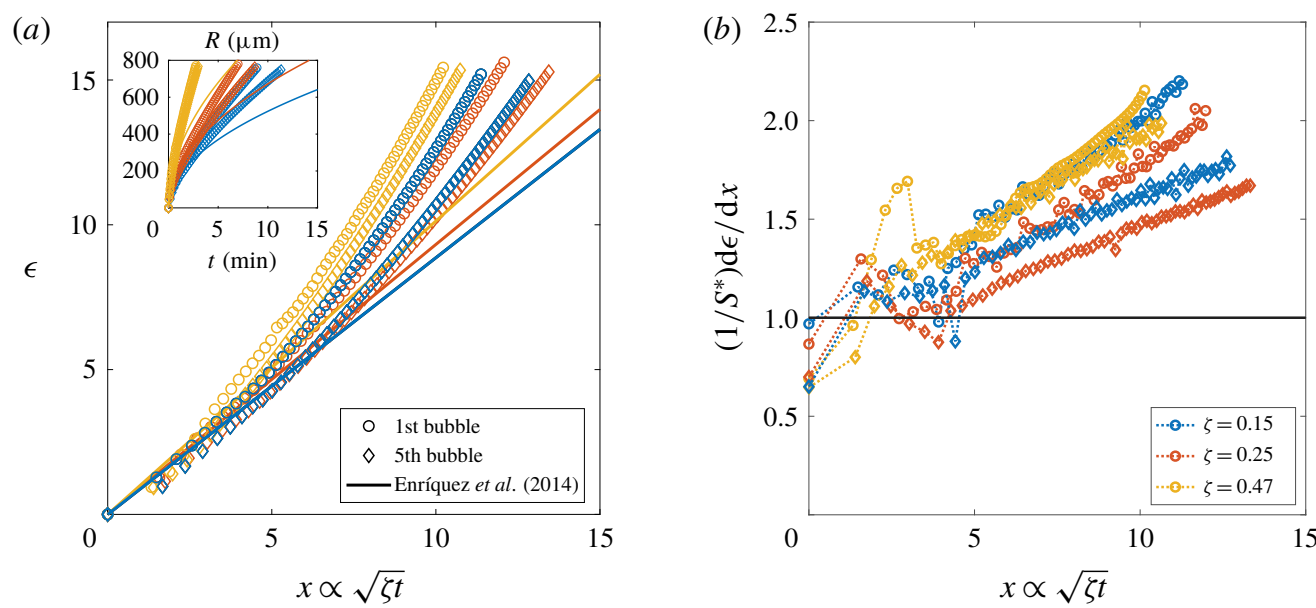

FIgURE 2. (Colour online) (a) Dimensionless radius $\epsilon$ plotted against the dimensionless square root of time $x$ for subsequent bubbles in confinement. The different colours represent different supersaturation levels $\zeta$, namely, blue for 0.15 , red for 0.25 and yellow for 0.47. The different symbols stand for the order of the bubble in the succession, namely circles for the first bubble and diamonds for the fifth, whereas (3.2) is plotted as solid lines in the corresponding colours. The inset shows the unscaled data for the time evolution of the bubble radius $R$ prior to the non-dimensionalisation. (b) Derivative of the dimensionless radius versus the dimensionless square root of time. The same colour symbol coding as in panel (a) applies here. Equation (3.2) coincides for the three different $\zeta$ and is represented as a black solid line. Note that increasing $\zeta$ causes an earlier and intensified onset of convection.

value, the faster it is expected that bubbles grow. We present the growth of different successive bubbles in confinement in figure $2(a)$. The bubble growth behaves as expected: higher supersaturation levels correspond to an increased bubble growth rate. The cases of $\zeta=0.15,0.25$ show very similar behaviour when plotted in their dimensionless form. Nonetheless, since $x \propto \zeta^{1 / 2}$, the unscaled data for smaller $\zeta$ lie below those of larger $\zeta$, as reflected in the inset of the same figure.

Depletion effects are expected to be strengthened by increasing the supersaturation level. The onset of density-driven convection permits the bubbles to absorb more gas than diffusion can supply from far away in the bulk solution, and specifically for the geometrical configuration regarded in this article (figure 1), the cylinder walls also play a crucial role in inhibiting the refreshment with non-depleted bulk liquid. In figure $2(b)$, we show the derivatives of the curves in figure $2(a)$ with respect to the dimensionless square root of time $x$. As the supersaturation increases, we can appreciate that the onset occurs earlier and is intensified, as indicated by a higher transient peak and a shorter plateau before the slope. We remind the reader that $x \propto \sqrt{\zeta t}$ in such a way that, even if the onset of convection seems to occur at a relative similar $x$, the absolute time is actually shorter for larger $\zeta$. Figure 3(a) represents the diverse values of $S h$ plotted against $R a$ for the different curves in figure 2. By comparing the experimental data to the theoretical relation (3.4) (which, as expected, lies above the experimental curves as it corresponds to a free bubble in an infinite medium), we distinguish a short early behaviour where $S h$ remains constant and indicates an early diffusive regime, and a transition to convection from the middle 

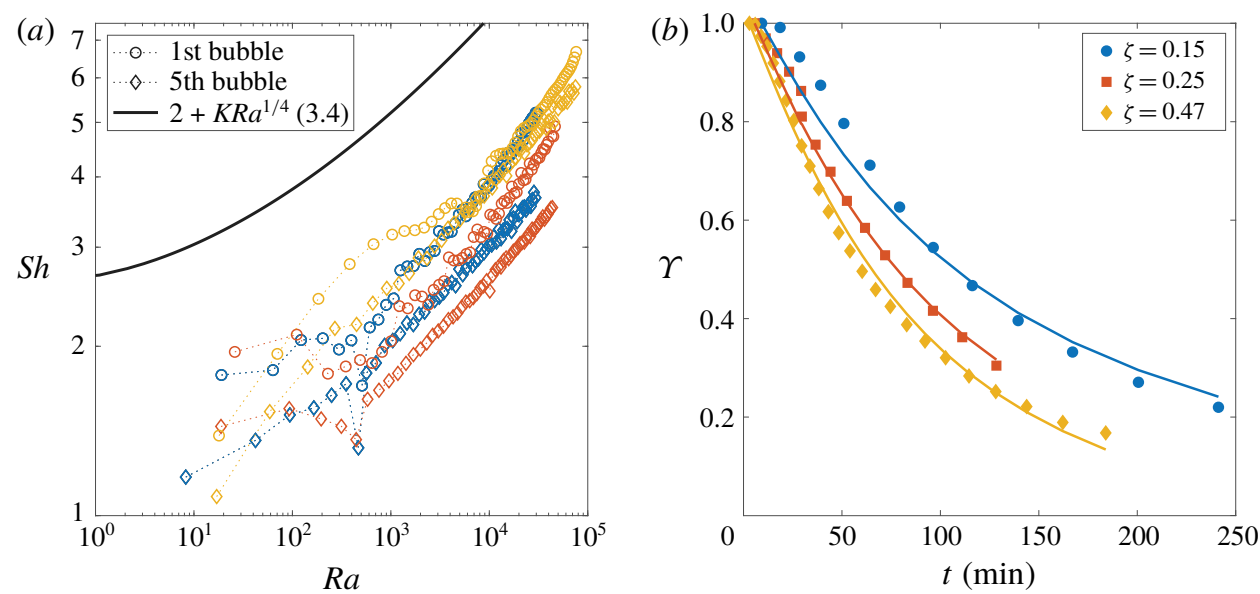

FIgURE 3. (Colour online) (a) Sherwood number $S h$ against Rayleigh number $R a$ for the same bubbles as in figure 2 plotted in logarithmic scale. Depletion can already be appreciated for the fifth bubble, which is clearly separated from the first curve. To compare, the theoretical relation for natural convection around bubbles in an infinite medium, equation (3.4), is shown. The experimental curves lie below but 'parallel' (see main text) to the theoretical expression, indicating that, indeed, natural convection is driving the bubble growth from the middle stages of its growth onwards. $(b)$ The depletion number $\Upsilon$ decreases for increasing $\zeta$, as expected from an enhanced bubble growth rate. The solid lines represent the best theoretical approximation for each $\zeta$ according to (3.7).

stages of the bubble growth, which has been also confirmed for the analogous case of droplets (Dietrich et al. 2016). The experimental curves are parallel to the theoretical one in the sense that was introduced in Moreno Soto et al. (2017), where it was shown that they collapse onto the same universal curve when they are rescaled using the depletion parameter $\Upsilon$ defined in (3.6), as we discussed in $\S 3$.

Regarding the long-term depletion effect, the different curves standing for the analytical solution (3.7) can be found in figure $3(b)$, together with the experimental results measured from collapsing the curves in figure 3(a). Similarly to the case of bubbles growing on an unconfined surface (Moreno Soto et al. 2017), $\Upsilon_{n}$ decreases for every subsequent bubble in the succession and, for the different curves, an increase in $\zeta$ implies a stronger depletion, i.e. $\Upsilon$ decreases faster for higher $\zeta$. The experiments are well described with the theoretical expression (3.7), with fitting constant $\alpha=0.57,0.53$ and 0.45 for $\zeta=0.15,0.25$ and 0.47 , respectively. The expected decreasing value of $\alpha$ with increasing $\zeta$ is also confirmed in the case of confinement. Note that $\Upsilon$ decreases faster in time for higher $\zeta$, but if we compare $\Upsilon$ for the same bubble number $n$, its value is bigger for larger $\zeta$. This is due to the longer times bubble need to grow until detachment for a smaller supersaturation. Therefore, convection has more time to develop such that gas diffusion from the non-depleted bulk cannot efficiently compensate for the absorbed gas, even though the onset of density-driven convection may be weaker as compared to higher supersaturated scenarios. The slight deviation between experiments and the theoretical fit may be connected to the one-dimensional (1-D) approximation. Moreover, the model seems to have difficulties capturing the behaviour of the first few bubbles in the succession. This also occurs for the experiments to be presented in the following 

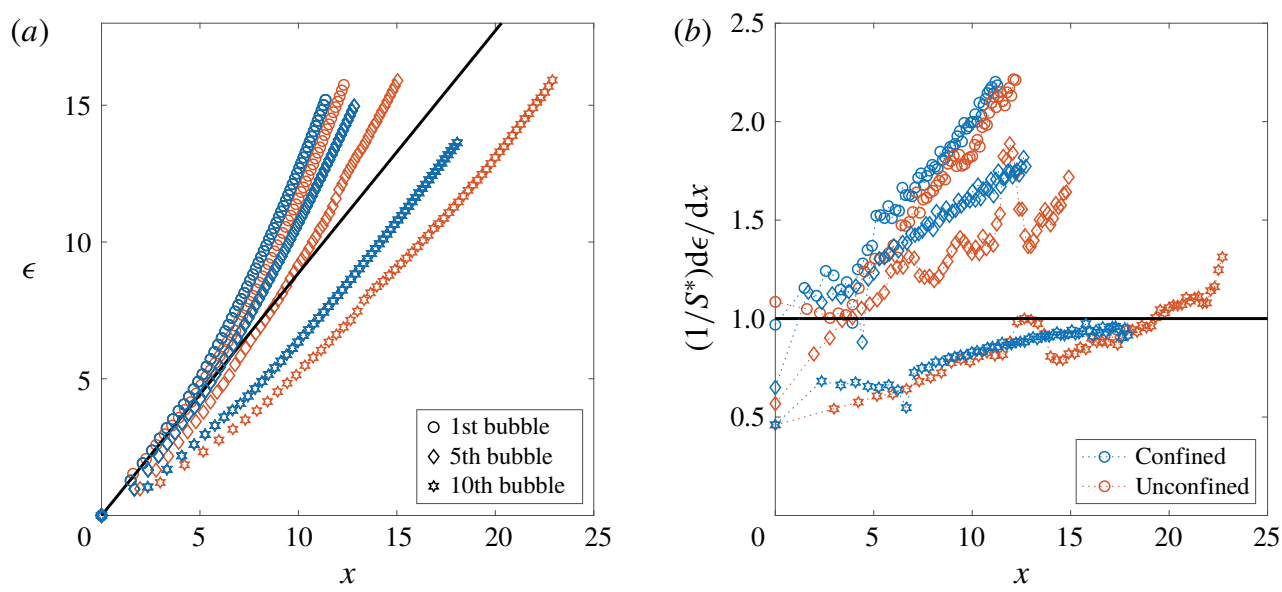

FIgURE 4. (Colour online) (a) Dimensionless radius $\epsilon$ plotted against the dimensionless square root of time $x$ for experiments with $\zeta=0.15$. In blue, the bubbles grow under confinement, whereas in red, the bubbles grow in an open medium. Different symbols are used to indicate the first (circle), fifth (diamond) and tenth (star) bubbles in the succession. The curves within the same succession deviate from each other due to the effect of depletion. $(b)$ Derivative of the dimensionless radius versus the dimensionless square root of time. The effects of depletion are very noticeable in this data representation. The respective curves with and without confinement seem to run parallel, indicating a similar strength in the onset of convection. In $(a, b)$, the solid line corresponds to (3.2).

section. For these first bubbles, the growing depleted concentration boundary layer has probably not yet reached the confinement walls and, therefore, the 1-D assumption is not yet applicable. We have indicated before that it takes up to $\approx 60 \mathrm{~min}$ for the concentration boundary layer to cover the full width of the cylinder. It is from that moment on that the 1-D model (3.7) is expected to capture the details of the effect of long-term depletion. As a closing remark, we stress that the three-dimensional (3-D) model in Moreno Soto et al. (2017) does not compare well to the experimental data presented in figure 3(b) for any value of $\alpha$. This supports the necessity of introducing a 1-D model to accurately describe the bubble behaviour in confinement.

To summarise this section, $\zeta$ has been proven to be the adequate control parameter which determines the speed at which bubbles grow, as well as an indicator of the speed and the intensity of depletion. The case is analogous to bubbles growing in a unbounded domain on a surface (Moreno Soto et al. 2017, 2019).

\section{Comparison of confined and unconfined bubble growth}

In this section we focus on the actual effect of the confinement by comparing with experiments in which the bubbles grow on a surface in an (for practical purposes) infinite medium. We discuss two sets of $\zeta$ independently, since, contrary to expectations, we will see that depletion evolves in a qualitatively different manner for each case.

\subsection{Supersaturation level $\zeta=0.15$}

The very low supersaturation level $\zeta=0.15$ corresponds to an extremely slow growth rate, approximately $20 \mathrm{~min}$ for the first bubbles. Figure 4(a) shows the dimensionless 
bubble radius evolution in dimensionless square root of time in confinement compared to the same in the unconfined case. The beginning of the bubble growth in both scenarios is very similar: bubbles grow according to the theoretical curve (3.2). However, and contrary to expectations, the bubbles under confinement seem to grow slightly faster than the bubbles in the open medium. This fact can be better appreciated in figure $4(b)$, where the derivatives show that the curves corresponding to the bubbles under confinement lie somewhat above those for the open medium. The transition to convection occurs slightly earlier for confined bubbles and consequently bubbles grow to some degree faster than those on an unconfined surface. This may originate from the local larger concentration gradients within the confined system, which would create a larger buoyant force on a smaller volume.

The depletion effects can be appreciated in figure $6(a)$, where $\Upsilon_{n}$ is plotted versus time. For a small supersaturation, depletion occurs faster in the confined case as a result of the rather enhanced bubble growth and the shielding effect of the walls for renewing the depleted liquid with fresh non-depleted solution once a bubble detaches. In the confined case, the mixing that occurs at detachment happens for this small supersaturation in a large boundary layer with a small concentration gradient, and therefore, its effect is less pronounced as compared to the supply of fresh, non-depleted solution in the unconfined scenario. In figure 6(a) we plot the theoretical model (3.7) for the confined and the one by Moreno Soto et al. (2017) for the unconfined case together with the corresponding experimental measurements, showing a good agreement for both models. Both the theoretical curves and the experiments show a very similar behaviour for the first few bubbles in the succession. This can be associated with the growth of the boundary layer, which for the confined case does not reach the walls of the cylinder until later in the succession. From that moment on, the curves start to diverge and hence, in the long term, depletion is slightly stronger in confinement than in the open system, as one would expect.

\subsection{Supersaturation levels $\zeta=0.25$ and 0.47}

Once the supersaturation $\zeta$ increases, confinement starts playing a major role on the way bubbles grow from the hydrophobic pit. Similarly to the previous subsection, figure 5(a) shows the dimensionless radius $\epsilon$ evolution for $\zeta=0.47$ (for $\zeta=0.25$, the bubble behaviour is qualitatively the same). Now, this case shows a faster growth for the first bubbles in the unbounded case than for the confined case, contrary to what happened in the previous subsection with $\zeta=0.15$. It is particularly interesting to notice that successive bubbles in confinement grow according to (3.2) until convection sets in, whereas for the unconfined case, only the very first bubbles in the succession match the analytical behaviour at the very early stages. This is due to the strong onset of convection and consequent depletion which occurs at supersaturations $\zeta<1-2$ achieved from a very high initial pressure $P_{0}$ (Moreno Soto et al. 2019). The higher $\zeta$, the faster and the stronger this onset occurs, as can be determined from figure $5(b)$. This transition appears to be inhibited in the confined case, such that convection sets in later in time and without such a strength.

Consequently, figures $6(b)$ and $6(c)$ show that bubbles in an unbounded domain deplete much faster than bubbles in confinement, represented by a faster decreasing $\Upsilon_{n}$. One could expect the opposite nonetheless, since the confinement impedes the dissolved gas in the bulk diffusing towards the bubble and compensating for the absorbed gas, which indeed happens in the case of bubbles growing in an open medium. The explanation of this enhanced depletion in the open system resides in 
(a)

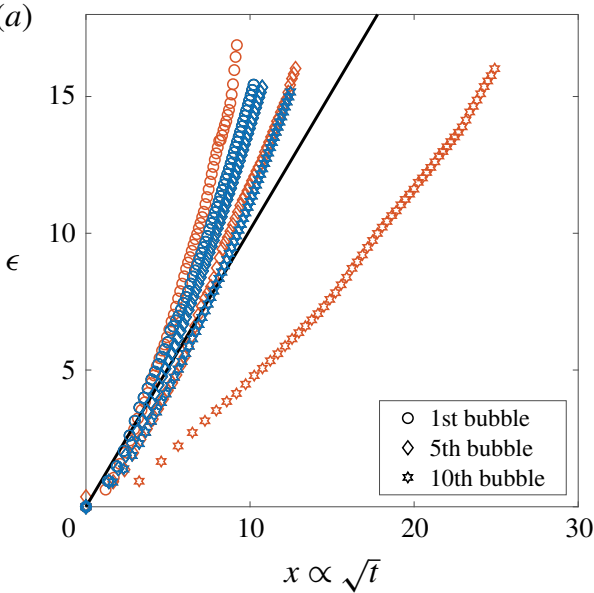

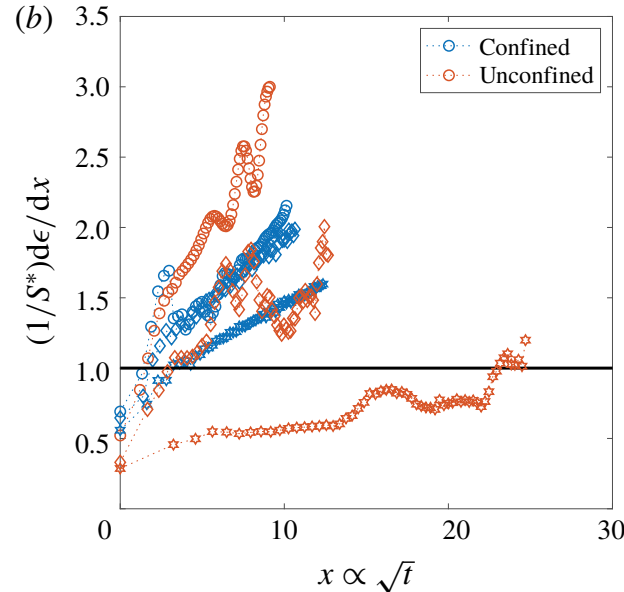

FIgURE 5. (Colour online) (a) Evolution of the dimensionless radius $\epsilon$ for $\zeta=0.47$ and (b) corresponding derivative versus the dimensionless square root of time $x$. The solid lines correspond to (3.2). There has been an inversion of some of the behaviour observed for $\zeta=0.15$ (figure 4): now, the first confined bubble grows slower than that in the unconfined case. However, due to a stronger depletion for the unbounded bubble, the subsequent bubbles in confinement grow faster.

the mechanism from which it originates: a full buoyant depleted volume is formed as bubbles absorb gas from their surroundings, which induces a stronger and intensified transition to natural convection. Note that the mass transfer enhancement is referred to the specific bubble diffusive growth and, therefore, the total mass transfer towards a bubble is always being reduced within the succession, equivalently to what occurs in figure $3(a)$. Consequently, bubbles progressively deviate more from the theoretical growth curve, equation (3.2), as the succession continues. That depleted volume rises together with the bubble after detachment and partially replaces the depleted solution with fresh liquid from the bulk (Moreno Soto et al. 2017). In bounded domains, these phenomena occur differently, since the confining walls firstly impede the formation of a buoyant depleted domain and, secondly, favour the gas mixing within the depleted area. These two effects combined result in a weaker long-term depletion. We note that convection has a slight effect on the final detachment radius (Enríquez et al. 2013), which also applies to the results in the previous section $\$ 5.1$. We have measured the ratio between the bubble radius at detachment for the confined and unconfined cases to be always larger than 0.9. However, this small difference can signify up to 10 min of additional growth time to achieve detachment. The accumulation of this time mismatch together with the larger depletion in the unconfined case continuously enlarge the lifetime of the bubbles, i.e. the bubble frequency decreases. Hence, figure 6 shows always a higher bubble frequency for the confined case, which is more noticeable for higher $\zeta$.

For time scales beyond the duration of our experiments, we anticipate that the impediment of solution refreshment due to the presence of the walls may start playing a dominant role and, therefore, the confined succession will start depleting faster as compared to the unbounded domain, as the cross-over by extrapolating the curves in figures $6(b)$ and $6(c)$ appears to suggest. 

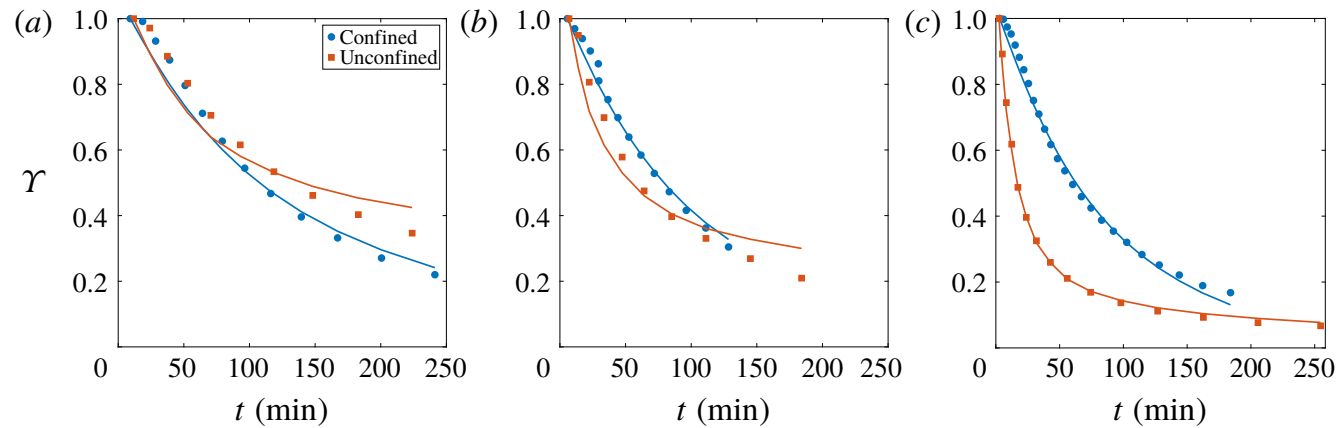

FIgURE 6. (Colour online) (a) The evolution of the depletion number $\Upsilon_{n}$ as a function of time for $\zeta=0.15$ shows that for the first five bubbles in the succession, bubbles in the confined and open cases deplete their surroundings in very similarly manner. In the long term, depletion is more affected by the minute stronger convective effect occurring in confinement. The solid lines in the corresponding colours represent the theoretical behaviour indicated by (3.7) for the confined domain (blue) and the model by Moreno Soto et al. (2017) for the non-confined (red). $(b, c)$ The depletion number $\Upsilon_{n}$ for $(b) \zeta=$ 0.25 and $(c) \zeta=0.47$ strongly decreases for the unconfined scenario as compared to the bounded domain due to the full development of the buoyant depleted boundary layer. Increasing $\zeta$ provides the same qualitative behaviour. Note that for all the cases, the bubble frequency for the confined case is always larger than for the unconfined case. This originates from the accumulation of time mismatch due to the slight difference in the final $R_{\text {det }}$ and the different ways in which the buoyant depleted boundary layer develops in the two cases.

\subsection{The cross-over between confined and unconfined bubble growth}

In the previous subsection, it was concluded that a very small supersaturation results in very similar behaviour for the confined and unconfined bubble growth, whereas for larger values the unbounded succession of bubbles depletes more strongly the solution in which they grow. However, looking at figures $6(b)$ and $6(c)$, it is justified to assume that there will be a cross-over time in which the impediment to the refreshment of the solution within the confinement becomes more influential than the partial evolution of the buoyant depleted boundary layer and the enhanced mixing of the solution.

By comparing the theoretical model (3.7) and the one in Moreno Soto et al. (2017), we have estimated that there may exist a cross-over time for the cases in which $\zeta \geqslant 0.17$. Lower values of the supersaturation level do not result in a cross-over between the two theoretical depletion models. Therefore, for $\zeta<0.17$, the confined bubble succession always depletes faster than the unbounded one, which corresponds to the results presented in $\S 5.1$ for $\zeta=0.15$. For the higher supersaturations, $\zeta=0.25$ and $\zeta=0.47$, we have predicted the cross-over times by extrapolating the theoretical curves shown in the corresponding figures $6(b)$ and $6(c)$, obtaining values of approximately 133 and $208 \mathrm{~min}$, respectively. These results qualitatively behave as expected: increasing $\zeta$ delays the cross-over time since the bubbles in the unconfined system undergo a much more intensified onset of density-driven convection and therefore deplete their surroundings much faster, whereas bubbles in confinement still suffer from the inhibiting effect of the walls on the evolution of the buoyant depleted boundary layer. We can therefore safely assume that, for $\zeta>0.17$, a cross-over time will always exist, at least provided that $\zeta$ is 
not so large that advective effects due to massive expansion of the bubble interface would set in and finally become dominant.

\section{Conclusions}

Confinement around bubbles causes major effects on their growth and the way in which they absorb gas from the surrounding liquid, since the walls act as a shield which prevents the renewal of the liquid contained within the confinement with fresh non-depleted liquid from the bulk. The supersaturation level $\zeta$ determines the speed at which bubbles grow in this configuration and how fast the available gas is extracted from the saturated liquid. The higher the supersaturation is, the earlier and more intensified the onset of natural convection occurs, which results in a faster depletion, analogously to what occurs with bubbles growing on an unconfined surface in an infinite bulk solution. For very low supersaturation levels, approximately $\zeta \approx 0.15$, confinement permits the bubble to grow slightly faster as compared to unbounded bubbles due to an enhanced onset of convection caused by the somewhat higher concentration gradients within the confined liquid. Therefore, the bubbles in confinement deplete somewhat faster their surroundings. Nonetheless, the evolution of confined and unconfined bubbles under very small supersaturation levels is rather similar. As the supersaturation is increased, the onset of natural convection plays a significant role from the early stages of the bubble growth. The origin of this convective onset plays a dominant role in the confined case, in which the cylinder walls impede the full formation of the buoyant gas-depleted region around the bubble. Besides, the confinement now enhances the mixing of the depleted liquid within the cylinder walls. Depletion effects consequently become weaker as compared to bubbles growing on an unconfined surface. Contrary to expectations, the lack of non-depleted liquid renewal from the bulk is counteracted by a better mixing within the confinement walls and a weaker onset of convection. On a much longer time scales, this compensating effect may actually be insufficient and the impediment to the refreshment of the solution with non-depleted bulk liquid in the confined case would start to cause a faster depletion at some point. The mathematical model (3.7) provides a good estimation of the long-term depletion effects in confinement despite its many simplifications. We expect that also with higher supersaturation levels $\zeta$, the model will still suffice as a first-order approximation to the results, but modifications will be necessary to account for many other side effects which originate at large $\zeta$, such as the appearance of parasitic bubbles which compete for the available gas and the much faster growth of the bubbles and their associated buoyant depleted boundary layer, which is expected to result in the onset of strong advective effects.

\section{Acknowledgements}

We thank R. Kooijman for the fabrication of the confinement cylinder on the substrates. This work was supported by the Netherlands Center for Multiscale Catalytic Energy Conversion (MCEC), an NWO Gravitation programme funded by the Ministry of Education, Culture and Science of the government of the Netherlands.

\section{Appendix A. One-dimensional model for the depletion number $\Upsilon_{n}$}

Confinement has an extensive effect on how bubbles deplete their surroundings by means of absorbing the dissolved gas from the liquid. As explained at the end of $\S 3$, a buoyant volume depleted of $\mathrm{CO}_{2}$ grows around the bubble and 
covers, in the intermediate term, the full width of the cylinder. The only available expansion direction is therefore along the vertical direction $z$, which corresponds to the longitudinal axis of the cylinder. The concentration boundary layer becomes much bigger than the bubble radius at detachment during the typical duration of our experiments, and therefore we can assume that the diffusive problem becomes one-dimensional in the $z$ direction.

To simplify matters, we model the succession of bubbles as a plane sink extracting gas from a one-dimensional domain along the longitudinal axis $z$. That way, we write the diffusive mass transfer equation as

$$
\frac{\partial \Delta c}{\partial t}=D \frac{\partial^{2} \Delta c}{\partial z^{2}}
$$

where $\Delta c=c-c_{0}$ in the longitudinal direction $z$. Two different boundary conditions and one initial condition are needed to obtain a unique solution for this equation, namely,

$$
\Delta c(z \rightarrow \infty, t) \rightarrow 0,\left.\quad D \frac{\partial \Delta c(z, t)}{\partial z}\right|_{z=0}=\frac{\dot{m}}{A_{c}}, \quad \Delta c(z, 0)=0 . \quad(\mathrm{A} 2 a-c)
$$

These boundary conditions correspond to replacing the complex processes of bubble growth and detachment with a diffusive problem observed from a more remote point of view. In that case, the first condition refers to the unalterability of the bulk concentration far away from the plane sink at all times. The second conditions models the diffusive flux at the plane sink as the average $\mathrm{CO}_{2}$ mass that is transported away by bubble detachment divided by the cross-section of the cylindrical confinement. Here, $\dot{m}=(4 / 3) \pi \rho_{g} R_{d e t}^{3} / t_{d e t}$ is the average mass transfer rate towards the growing bubble until its detachment and $A_{c}=\pi R_{c}^{2}$ is the cross-sectional area of the confinement. If we relate these two parameters, we obtain $\dot{m} / A_{c}=4 \rho_{g} R_{\text {det }}^{3} / 3 R_{c}^{2} t_{\text {det }}$. The third condition corresponds to the initial state of the environment, which consists of a uniformly supersaturated bulk liquid. As standard for heat transfer problems (see e.g. Bejan 1993), this partial derivative equation is solved by means of Laplace transforms, in which we take the function $\Delta c(z, t)$ from a temporal domain $t$ to a frequency domain $s: \mathcal{L}\{\Delta c(z, t)\}(s)=\mathcal{C}(z, s)$. We can then reformulate (A 1) and write

$$
\frac{\partial^{2} \mathcal{C}(z, s)}{\partial z^{2}}=\frac{1}{D} \mathcal{L}\left\{\frac{\partial \Delta(z, t)}{\partial t}\right\}(s)=\frac{1}{D}(s \mathcal{C}(z, s)-\Delta c(z, 0)) .
$$

By imposing the initial condition, it follows that

$$
\frac{\partial^{2} \mathcal{C}(z, s)}{\partial z^{2}}=\frac{s}{D} \mathcal{C}(z, s),
$$

which can be directly integrated to obtain a solution of the type

$$
\mathcal{C}(z, s)=A \exp \left(-\sqrt{\frac{s}{D}} z\right)+B \exp \left(\sqrt{\frac{s}{D}} z\right) .
$$

To determine the values of the integral constants, we need to impose the two spatial boundary conditions once transformed to the frequency domain $s$. By applying the first one,

$$
\mathcal{L}\{\Delta c(z \rightarrow \infty, t)\}(s)=\mathcal{C}(\infty, s)=0,
$$


we directly obtain that $B=0$. By the second one,

$$
\mathcal{L}\left\{\left.D \frac{\partial \Delta c(z, t)}{\partial z}\right|_{z=0}\right\}(s)=\left.D \frac{\partial \mathcal{C}(z, s)}{\partial z}\right|_{z=0}=\mathcal{L}\left\{\frac{\dot{m}}{A_{c}}\right\}(s)=\frac{\dot{m}}{s A_{c}},
$$

we calculate $A$. The final solution in the frequency domain reads

$$
\mathcal{C}(z, s)=-\frac{\dot{m}}{A_{c} D} \frac{1}{s} \sqrt{\frac{D}{s}} \exp \left(-\sqrt{\frac{s}{D}} z\right),
$$

which transformed back to the temporal domain becomes

$$
\Delta c(z, t)=-\frac{\dot{m}}{A_{c} D}\left(2 \sqrt{\frac{D t}{\pi}} \exp \left(-\frac{z^{2}}{4 D t}\right)-z \operatorname{erfc}\left(\frac{z}{2 \sqrt{D t}}\right)\right) .
$$

The variation of the depletion number $\Delta \Upsilon_{n}=\Upsilon_{n}-\Upsilon_{n-1}$ is obtained by dividing $\Delta c$ (A 9) by $\left(c_{0}-c_{s}\right)$. The 1-D length of the concentration boundary layer at detachment can be written as $R_{d e t}+\alpha \sqrt{3.3 D t_{d e t}}$. Here, $\alpha$ is a fitting constant as defined in (3.7) and relates to the distance within the concentration boundary layer that sets the apparent concentration for the next bubble in the succession. The prefactor 3.3 is included because it determines the point at which the concentration difference within the boundary layer reaches $0.99\left(c_{0}-c_{s}\right)$ following a erfc distribution. We point out that this definition corresponds to an 'effective' boundary layer thickness, such that $\alpha$ represents a fraction of it. By substituting the distance $z$ in (A 9) by the length of the concentration boundary layer, we obtain the final expression for the depletion parameter (3.7):

$$
\begin{aligned}
\Upsilon_{n}= & \Upsilon_{n-1}-\frac{4 \rho_{g} R_{d e t}^{3}}{3 R_{c}^{2} t_{d e t} D\left(c_{0}-c_{s}\right)}\left[2 \sqrt{\frac{D t_{d e t}}{\pi}} \exp \left(-\frac{\left(R_{d e t}+\alpha \sqrt{3.3 D t_{d e t}}\right)^{2}}{4 D t_{d e t}}\right)\right. \\
& \left.-\left(R_{d e t}+\alpha \sqrt{3.3 D t_{d e t}}\right) \operatorname{erfc}\left(\frac{R_{d e t}+\alpha \sqrt{3.3 D t_{d e t}}}{2 \sqrt{D t_{d e t}}}\right)\right]
\end{aligned}
$$

Note that although this model corresponds to the solution of a pure diffusive bubble growth, the onset of density-driven convection is accounted for in the fitting parameter $\alpha$ and the experimental detachment time $t_{d e t}$ (which increases for each subsequent bubble and therefore results in a larger concentration boundary layer upon detachment).

\section{REFERENCES}

Ajaev, V. S. \& Homsy, G. M. 2006 Modeling shapes and dynamics of confined bubbles. Annu. Rev. Fluid Mech. 38, 277-307.

Bao, B., Zandavi, S. H., Li, H., Zhong, J., Jatukaran, A., Mostowfi, F. \& Sinton, D. 2017 Bubble nucleation and growth in nanochannels. Phys. Chem. Chem. Phys. 29 (12), 8223-8229.

Barber, J., Brutin, D., Sefiane, K. \& TAdrist, L. 2010 Bubble confinement in flow boiling of FC-72 in a 'rectangular' microchannel of high aspect ratio. Exp. Therm. Fluid Sci. 34 (8), $1375-1388$.

BeJAn, A. 1993 Heat Transfer, 1st edn. John Wiley \& Sons. 
Bergman, T. L., Incropera, F. P., DeWitt, D. P. \& Lavine, A. S. 2011 Fundamentals of Heat and Mass Transfer, 7th edn. John Willey \& Sons.

Borkent, B. M., Gekle, S., Prosperetti, A. \& Lohse, D. 2009 Nucleation threshold and deactivation mechanisms of nanoscopic cavitation nuclei. Phys. Fluids 21 (102003), 1-9.

Clift, R., Grace, J. R. \& Weber, M. E. 1978 Bubbles, Drops and Particles, United Kingdom edn. Academic Press.

Dietrich, E., Wildeman, S., Visser, C. W., Hofhuis, K., Kooij, E. S., Zandvliet, H. J. W. \& LOHSE, D. 2016 Role of natural convection in the dissolution of sessile droplets. J. Fluid Mech. 794, 45-67.

Enríquez, O. R., Hummelink, C., Bruggert, G.-W., Lohse, D., Prosperetti, A., van der MeER, D. \& Sun, C. 2013 Growing bubbles in a slightly supersaturated liquid solution. Rev. Sci. Instrum. 84, 065111.

Enríquez, O. R., Sun, C., Lohse, D., Prosperetti, A. \& Van der Meer, D. 2014 The quasi-static growth of $\mathrm{CO}_{2}$ bubbles. J. Fluid Mech. 741, R1.

Epstein, P. S. \& Plesset, M. S. 1950 On the stability of gas bubbles in liquid-gas solutions. J. Chem. Phys. 18 (11), 1505-1509.

Evans, G. M. \& Machniewski, P. M. 1999 Mass transfer in a confined plunging liquid jet bubble column. Chem. Engng Sci. 54 (21), 4981-4990.

Fritz, W. 1935 Berechnung des Maximal Volume von Dampfblasen. Phys. Z. 36, 379-388.

Gedupudi, S., Zu, Y. Q., Karayiannis, T. G., Kenning, D. B. R. \& Yan, Y. Y. 2011 Confined bubble growth during flow boiling in a mini/micro-channel of rectangular cross-section. Part I: Experiments and 1-D modelling. Intl J. Therm. Sci. 50 (3), 250-266.

Hong, S., TANG, Y., LAI, Y., WANG, S. \& ZhANG, L. 2016 Visualization research on confined bubble growth feature and geat transfer characteristic in ultra-shallow micro channel. Intl $J$. Heat Mass Transfer 103, 847-854.

Huisman, S. G., van Gils, D. P. M. \& Sun, C. 2012 Applying laser Doppler anemometry inside a Taylor-Couette geometry using a ray-tracer to correct for curvature effects. Eur. J. Mech. (B/Fluids) 36, 115-119.

Leighton, T. G. 2011 The inertial terms in equations of motion for bubbles in tubular vessels or between plates. J. Acoust. Soc. Am. 130 (5), 3333-3338.

Liu, X., Coxon, P. R., Peters, M., Hoex, B., Cole, J. M. \& Fray, D. J. 2014 Black silicon: fabrication methods, properties and solar energy applications. Energy Environ. Sci. 7 (10), 3223-3263.

Moreno Soto, Á, Enríquez, O. R., Prosperetti, A., Lohse, D. \& VAn Der Meer, D. 2019 Transition to convection in single bubble diffusive growth. J. Fluid Mech. 871, 332-349.

Moreno Soto, Á, Prosperetti, A., Lohse, D. \& VAn der Meer, D. 2017 Gas depletion through single gas bubble diffusive growth and its effect on subsequent bubbles. J. Fluid Mech. 831, 474-490.

MukherJee, A., Kandlikar, S. G. \& Edel, Z. J. 2011 Numerical study of bubble growth and wall heat transfer during flow boiling in a microchannel. Intl J. Heat Mass Transfer 54 (15-16), 3702-3718.

Oehmichen, T., Datsevich, L. \& Jess, A. 2010 Influence of bubble evolution on the effective kinetics of heterogeneously catalysed gas/liquid reactions. Part I. Reactions with gaseous products. Chem. Engng Technol. 33 (6), 911-920.

OG̃UZ, H. N. \& Prosperetti, A. 1993 Dynamics of bubble growth and detachment from a needle. J. Fluid Mech. 257, 111-145.

RAHMAN, S. U. 2013 Natural convection transport from sphere held in vertical cylindrical cavities. Arab. J. Sci. Engng 38 (8), 1951-1957.

Rivero-Rodríguez, J. \& SCHEID, B. 2019 Mass transfer around bubbles flowing in cylindrical microchannels. J. Fluid Mech. 869, 110-142.

Sillen, C. W. M. P., Barendrecht, E., Janssen, L. J. J. \& Van Stralen, S. J. D. 1982 Gas bubble behaviour during water electrolysis. Intl J. Hydrogen Energy 7 (7), 577-587.

Somorjai, G. A. \& Li, Y. 2010 Introduction to Surface Chemistry and Catalysis, 2nd edn. John Willey \& Sons. 
Stubenrauch, M., Fischer, M., Kremin, C., Stoebenau, S. \& Albrecht, A. 2006 Black silicon - new functionalities in microsystems. J. Micromech. Microengng 16 (6), S82-S87.

Verhaart, H. F. A., De Jonge, R. M. \& van Stralen, S. J. D. 1980 Growth rate of a gas bubble during electrolysis in supersaturated liquid. Intl J. Heat Mass Transfer 23 (3), 293-299.

Vincent, O. \& Marmottant, P. 2017 On the statics and dynamics of fully confined bubbles. J. Fluid Mech. 827, 194-224.

YIN, L. \& JIA, L. 2016 Confined bubble growth and heat transfer characteristics during flow boiling in microchannel. Intl J. Heat Mass Transfer 98, 114-123.

Zu, Y. Q., Yan, Y. Y., Gedupudi, S., Karayiannis, T. G. \& Kenning, D. B. R. 2011 Confined bubble growth during flow boiling in a mini-/micro-channel of rectangular cross-section. Part II. Approximate 3-D numerical simulation. Intl J. Therm. Sci. 50 (3), 267-273. 